\title{
Some variables affecting feeding cycles in the opossum
}

\author{
W. F. ANGERMEIER \\ Universität zu Köln, Köln, Federal Republic of Germany \\ and \\ J. MCLEAN and D. MINVIELLE \\ University of New Orleans, New Orleans, Louisiana
}

\begin{abstract}
The feeding behavior of 4 wild male opossums (Didelphis virginiana) was measured under four different conditions, keeping the light:dark (L:D) cycle constant at 12:12. These conditions were: (1) L:D cycle synchronized with sunrise and sunset $(D=18: 00-06: 00, L=06: 00-18: 00) ;(2) \mathrm{L}: D$ cycle shifted from $\mathrm{D}=18: 00-06: 00$ to $\mathrm{D}=14: 00-02: 00$ (a 1-h daily shift over the next 4 days); (3) L:D cycle held steady: $D=14: 00-02: 00$; and (4) L:D cycle held steady as in Condition 3 with $50 \%$ reduced availability of food. As compared to Condition 1, all other conditions produced a major shift of feeding behavior toward the beginning of the dark cycle.
\end{abstract}

A number of investigators have studied the effects of the light-dark (LD) cycle upon activity (Ashby, 1972; D. M. Cone, 1973; D. M. Cone \& A. L. Cone, 1968) and upon feeding and drinking behavior (Rusak \& Zucker, 1974; Stephan, 1984). Such studies attain great importance, particularly when one considers the implications of their results on learning. Holloway and Wansley (1973) were able to show that retention of active and passive avoidance behavior is fairly good at $12-\mathrm{h}$ multiple intervals following training and very poor at intermediate times. The study reported here sought to investigate several variables that might affect feeding rhythms in the opossum, such as shifts of the L:D 12:12 cycle to an earlier time and reduction of the availability of food. The investigation was conducted preparatory to food-rewarded operant learning in the opossum.

\section{METHOD}

\section{Subjects}

Four wild, male, 9-month-old Virginia opossums (Didelphis virginiana) were used as subjects. They were obtained from a licensed dealer and kept singly in stainless-steel cages $(90 \times 60 \times 60 \mathrm{~cm}$ high) which also contained a nesting box $(60 \times 30 \times 12 \mathrm{~cm}$ high) and a resting perch $(60 \times 20 \mathrm{~cm}$ wide). The cage was filled with wood shavings and finely shredded paper. The resting perch was $40 \mathrm{~cm}$ off the floor on the right side of the cage. Commercial dog food and water were available ad lib, except when stated otherwise. Placement of food always occurred at the same time, that is, $2 \mathrm{~h}$ before the dark cycle began. Once a week each subject received $100 \mathrm{~g}$ of meat.

This research was supported in part by a grant from the Deutsche Forschungsgemeinschaft (DFG) Bonn, Federal Republic of Germany, and by the Department of Psychology, The University of New Orleans, New Orleans, LA.

Requests for reprints should be sent to the senior author at: Psychologisches Institut I, Lehrstuhl II: Angermeier, Universität zu Köln, Meister-Ekkehart-Str. 9, 5000 Köln 41, Federal Republic of Germany (BRD).

\section{Apparatus}

Data were collected in the animals' home cages. Each cage was wired so that when an animal made contact with the food cup, it completed a circuit between the cup and the cage. This information was registered by a computer in terms of real time (hours-minutes-seconds).

\section{Procedure}

Under Condition 1, an L:D 12:12 cycle was in effect, with the dark period extending (roughly synchronized with sunset and sunrise) from 18:00 to 06:00 and the light period lasting from 06:00 to 18:00. Ad-lib food and water were made available $2 \mathrm{~h}$ before the dark cycle began. For all conditions, the cages were cleaned daily and the animals were handled for 3-5 min $3 \mathrm{~h}$ before the dark cycle began. All conditions were in effect for at least 4 days.

Under Condition 2, an L:D 12:12 cycle was in effect, but was shifted forward 1 h daily for 4 days as follows: on Day 1 the dark cycle lasted from 17:00 to 05:00; on Day 2 from 16:00 to 04:00; and so forth.

Condition $3 \mathrm{kept}$ the L:D 12:12 cycle constant with the dark period beginning at 14:00 and ending at 02:00.

Condition 4 was the same as Condition 3, except that available food was reduced to $50 \%$ of the individual animal's food consumption during the previous 15 days.

\section{RESULTS AND DISCUSSION}

The results of Conditions 1 and 2 are compared in Figure 1. Note that responses to the food cup began shortly after the beginning of the dark cycle, 18:00, and continued throughout, reaching a peak at approximately 01:00. Also note that in all four conditions there was a virtual absence of food responses during light phases. Figure 1 indicates a shift of the peaks in the percentages of approaches to the food cups in the direction of the beginning of the dark cycle. The shift, however, is incomplete.

The results of Conditions 1 and 3 are compared in Figure 2. As is evident in the figure, moving the start of the dark cycle to 02:00 resulted in a major shift of the percentages in the approach to the food cups toward the beginning of the dark cycle. In Condition 1 major feed- 
ing activity shifted from zero to medium to high, whereas in Condition 3 the feeding pattern was inverted, running from high to medium and finally to zero. In essence, shifting the dark cycle to $14: 00$ produced a mirror-image of Condition 1.

As shown in Figure 3, there was a slight increase in responses to the food cup before the dark cycle started, that is, between 12:00 and 14:00 $\mathrm{h}$. There was also a rapid decline of activity taking place after the second peak at 19:00 h.

The feeding behavior of the wild opossum seems to be heavily influenced by the onset of dark. This observation

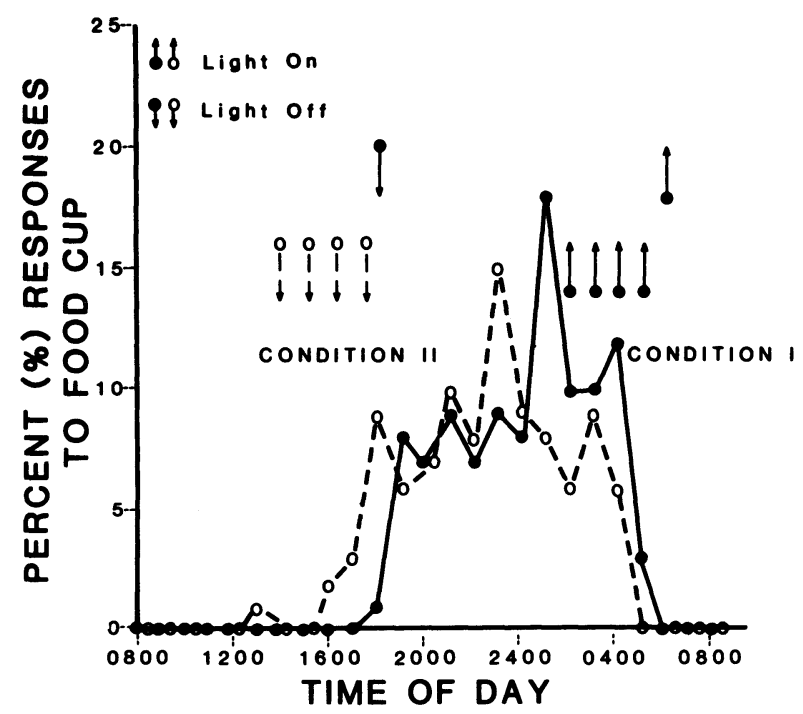

Figure 1. Comparison of Conditions 1 and 2. Condition 1 had an L:D 12:12 cycle, beginning at 18:00 and ending at 06:00. In Condition 2 the L:D 12:12 cycle was shifted $1 \mathrm{~h}$ daily.

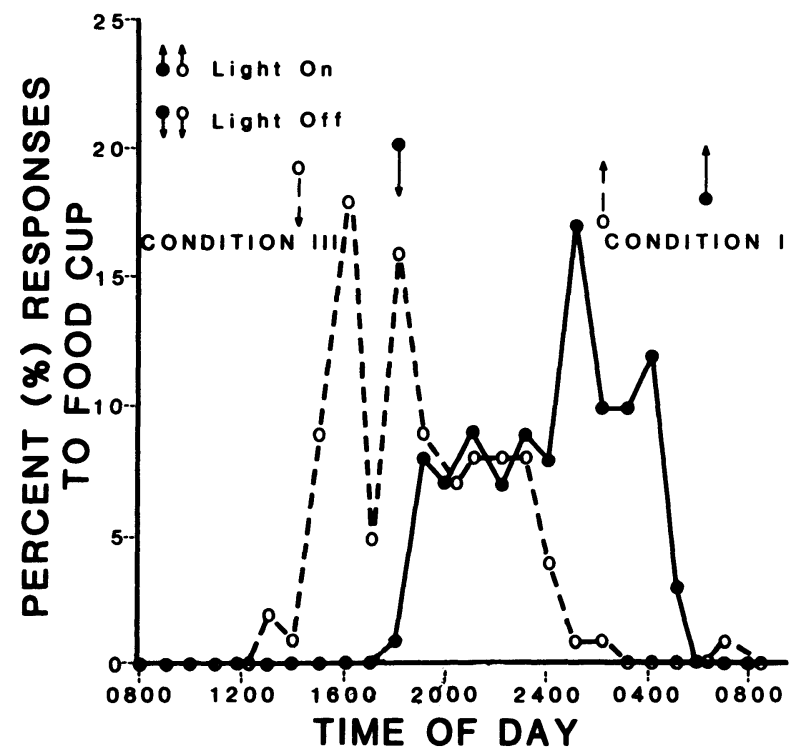

Figure 2. Comparison of Conditions 1 and 3 with L:D 12:12 cycles beginning and ending at 18:00 and 06:00 or 14:00 and 02:00 $h$ respectively.

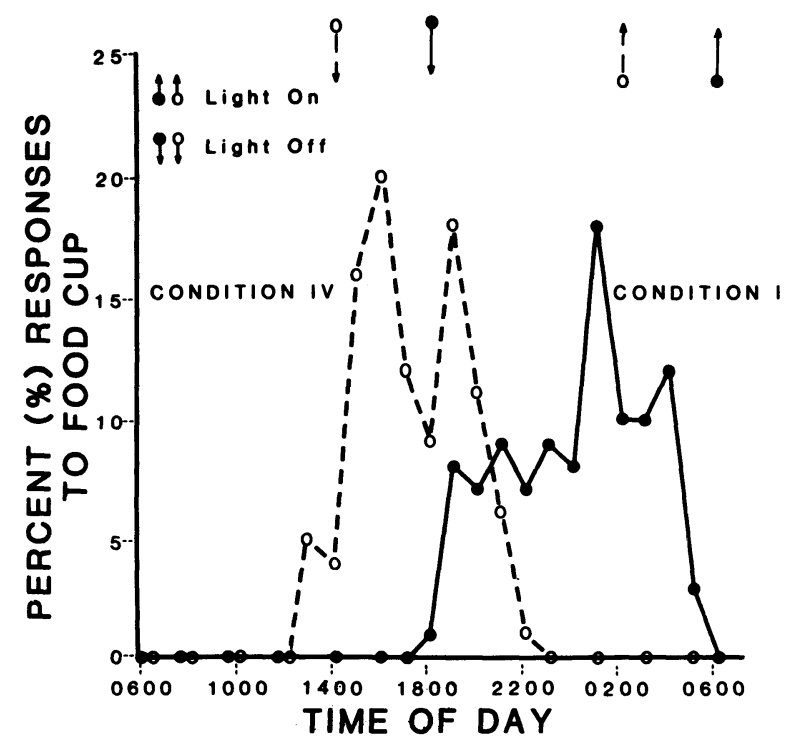

Figure 3. Comparison of Conditions 1 and 4. Condition 4 is the same as Condition 3, with food reduced to $50 \%$ of the average amount consumed during a previous 15-day period of ad-lib feeding.

is confirmed by the data presented here. When the onset of dark is shifted forward in time, the responses of the food cup accompany that shift. This is seen most clearly in Figure 2 and agrees with the findings of other investigators (Aschoff, 1984; Terman, Gibbon, Faihurst, \& Waring, 1984). In addition, reduced availability of food shortens the length of the total period of food-getting activity by $2-3$ hours (Figure 3 ). Normally $50 \%$ of all foodgetting activity occurs 6-10 $\mathrm{h}$ after the onset of dark (Condition 1$)$, whereas nearly that much $(48 \%)$ occurs within $3 \mathrm{~h}$ after dark (Condition 4).

In the rat, anticipation of food access is mediated by a circadian mechanism several hours before feeding (Stephan, 1984). It would be interesting to speculate whether this is also true for the opossum and perhaps partially or wholly responsible for the results obtained here.

These observations indicate rapid extinction of approaching the empty food cups. The temporal shifts are also accomplished in a relatively short period of time, a fact that seems to underline the survival advantage of such behavior in one of the oldest mammals surviving to this day (Eisenberg, 1981).

\section{REFERENCES}

Aschoff, J. (1984). Circadian timing. Annals of New York Academy of Science, 423, 442-468.

Ashby, K. R., (1972). Patterns of daily activity in mammals. Mammalian Review, 1, 171-185.

Cone, D. M. (1973). Circadian locomotor activity in the Virginia opossum. Bulletin of the Psychonomic Society, 1, 13-14.

Cone, D. M., \& Cone, A. L. (1968). Cage activity in the Virginia opossum. Psychonomic Science, 10, 259-260.

EISENBERG, J. F. (1981). Mammalian radiations (pp. 20-22). Chicago: The University of Chicago Press.

Holloway, F. A., \& Wansley, R. A. (1973). Multiphasic retention 
deficits at periodic intervals after passive avoidance learning. Science, 180, 208-210.

RUSAK, B., \& ZUCKER, I. (1974). Fluid intake of rats in constant light and during feeding restricted to the light or dark portion of the illumination cycle. Physiology \& Behavior, 13, 91-100.

StePhan, F. K. (1984). Phase shifts of circadian rhythm in activity entrained to food access. Physiology \& Behavior, 32, 663-671.
Terman, M., Gibbon, J., Faihurst, S., \& Waring, A. (1984). Daily meal anticipation: Interaction of circadian and interval timing. Annals of New York Academy of Science, 423, 470-487.

(Manuscript received for publication July 12, 1986.) 